\title{
Discrete approximation of nonlinear control problems
}

\author{
Riho Lepp \\ Institute of Cybernetics, Estonian Academy of Sciences \\ Akadeemia 21, EE-0026 Tallinn, Estonia. \\ Tel: +3722-527902. Fax: +3722-527901. \\ e-mail: Iprh@ioc.ee
}

\begin{abstract}
The basic optimal control problem with bounded measurable controls and absolutely continuous trajectories is approximated by a sequence of finite-dimensional problems. Using the notion of the discrete convergence of elements and operators, conditions are presented that quarantee the discrete convergence of trajectories and the weak* discrete convergence of controls.
\end{abstract}

\section{Keywords}

Nonlinear control equation, bounded measurable controls, discrete convergence

\section{INTRODUCTION}

Consider the following basic optimal control problem (Daniel, 1973): Minimize

$f(x, u)=\int_{0}^{T} f(t, x(t), u(t)) d t$,

over the set of functions $\{(x(t), u(t))\}$ satisfying the differential equation

$\dot{x}(t)=g(t, x(t), u(t))$ for almost all (a.a.) $t \in[0, T], x(0)=x_{0}$,

and the additional control constraints

$u(t) \in U_{a d}$ for a.a. $t \in[0, T]$,

where functions $f(t, x, u)$ and $g(t, x, u)$ are nonlinear in all variables $(t, x, u), f: R \times$ $R^{r} \times R^{m} \rightarrow R, g: R \times R^{r} \times R^{m} \rightarrow R^{r}$.

In order to to write the problem as an optimization problem in a Banach space, we have to choose controls $u(t)$ as elements of the space of essentially bounded measurable functions, $u \in L^{\infty}([0, T])$, otherwise the functional (1) and operator (2) are not 
differentiable and thus, certain difficulties arise with formulation of the second order optimality conditions. The trajectories $x(t)$, corresponding to controls $u(t)$, are assumed to be either absolutely continuous (Daniel, 1973), $x \in W^{1,1}([0, T])$, or Lipschitz continuous, $x \in W^{1, \infty}([0, T])$ (Hager, 1990).

In practice, the minimization of control problems is carried out in finite-dimensional subspaces, replacing integrals by sums and infinite number of constraints by finite ones. Correctness of this replacement is usually guaranteed by systems of projectors $\left\{P_{n}\right\}$ such that

$\left\|P_{n} u-u\right\| \rightarrow 0, \quad n \in N, \forall u \in U_{a d}$

(e.g. by orthoprojectors in the Ritz-Galerkin method (Daniel, 1973)).

This approximation scheme works well in $L^{p}$-spaces, $1 \leq p<\infty$, and results, generally speaking, from the fact that in Lebesgue spaces we can approximate functions by polynomials with rational coefficients.

The situation changes drastically if controls belong to the space of essentially bounded measurable functions $L^{\infty}([0, T])$ since the space $C([0, T])$ of continuous functions is not dense there and thus, it is not clear, how to construct systems of subspaces and projectors which could satisfy (4). At the same time it is more convenient to formulate nonlinear control problems in $L^{\infty}$ since in $L^{p}$-spaces, $1 \leq p<\infty$, functions $f(t, x, u)$ and $g(t, x . u)$ should satisfy certain growth conditions and this in turn, together with the weak convergence of controls brings along linearity in $u$ of functions $f(t, x, u)$ and $g(t, x, u)$, and, as it was mentioned earlier, functional in (1) and operator in (2) what define the problem, are not differentiable in $L^{p}$ spaces.

Still, there exists at least one possibility to approximate nonlinear control problems in $L^{\infty}$. This possibility is based on the concept of the discrete convergence of elements and operators, and was formulated and developed in the 70 -ies by many authors in numerical analysis (see, e.g. , (Stummel, 1971), (Vainikko, 1978)). The main differences between the classical projection method and the new discretization method approaches are:

1) in the discretization approach $P_{n}$-s could not to be projectors since approximate problems need not to be formulated in subspaces;

2) in the discretization approach instead of strong convergence of projected elements (4) only consistency of norms between initial and approximate spaces is needed. This norm consistency condition guarantees the nondegeneracy of norms of the discretized elements and thus, uniqueness of the limit process.

The norm consistency condition is weaker than the convergence condition (4). For instance, in reflexive $L^{p}$-spaces, $1<p<\infty$, from the weak convergence of elements and from the convergence of their norms it follows the strong (norm) convergence of elements (the Radon - Riesz property).

The norm consistency condition between the space of essentially bounded functions $L^{\infty}$ and the sequence of Euclidean spaces with increasing dimension was formulated and proved in (Lepp, 1988) for a system of linear piecewise integral connection operators, where the partition of the integration domain was realized using only the sets with measure zero of their boundary. Note that for projectors $P_{n}$, defined in such way, it can happen that there exist $L^{\infty}$-functions for which the limit (4) does not exist (see, e.g. , (Atkinson, 1983)). 
In this paper we will follow the next scheme. Relying on the Banach fixed point theorem, we can show for fixed controls $u$ and $u_{n}$ that there exist trajectories $x$ and $x_{n}$ as solutions of initial and approximate equations. Since the set of admissible controls $U_{a d}$ is bounded, we can separate from a minimizing control sequence $\left\{u_{n}\right\}$ a weakly* discretely convergent subsequence. Then, showing that the approximate control equations are collectively compact (Anselone, 1971), we can guarantee the discrete convergence of trajectories. Finally, the convergence of approximate optimal controls and trajectories follows from the properties of the cost function.

Schemes of analysis of the stability of discrete approximations of infinite dimensional extremum problems rely on discrete analogues of the existence theorems (see, e.g. , (Daniel, 1969), (Vasin, 1982)). Hence, we at first formulate a theorem what gives us the existence of an optimal solution in $C([0, T]) \times L^{\infty}([0, T])$. Due to technical reasons we can not guarantee compactness in $x$ of the integral operator $g(x, u)=\int_{0}^{t} g(s, x(s), u(s)) d s$ in $W^{1,1}([0, T])$, but only in $C([0, T])$ for a fixed $u \in U_{a d}$. Consequently, convergence analysis of trajectories is carried out in a space with weaker topology than the topology of the space of absolute continuous functions.

Since the existence problems for differential equations are more convenient to handle in their integral form, we will reformulate the equation (2) as an integral equation:

$x(t)=\int_{0}^{t} g(s, x(s), u(s)) d s+x_{0}, \quad t \in[0, T]$.

Assume that

f.1) the function $f(t, x, u)$ is Riemann integrable in $t$ for all $(x . u)$, continuously differentiable in $(x, u)$ for a.a. $t$ and for all $h>0$ and all $|x|,|y|,|u| \leq h$ there exist Riemann integrable functions $a_{1 h}(t), a_{2 h}(t)$, such that $|f(t, x, u)| \leq a_{1 h}(t)$ and $|f(t, x,, u)-f(t, y, u)| \leq a_{2 h}(t)|x-y|$

$f .2)$ the function $f_{u}^{\prime}(t, x, u)$ is Riemann integrable in $t$ for all $(x, u)$, and continuous in $(x, u)$ for a.a. $t \in[0, T]$, there exists a Riemann integrable function $a_{3 h}(t)$ such that $\left|f_{u}^{\prime}(t, x, u)\right| \leq a_{3 h}(t)$ for all $h>0,|x|,|u| \leq h$;

g.1) the function $g(s, x, u)$ is Riemann integrable in $s$ for all $(x, u)$, continuously differentiable in $(x, u)$ for a.a. $t$ and for all $h>0$ and all $|x|,|u|$ there exists a Riemann integrable function $b_{1 h}(s)$ such that $|g(s, x, u)| \leq b_{1 h}(s)$;

g.2) the function $g_{x}^{\prime}(s, x, u)$ is Riemann integrable in $s$ for all $(x, u)$, continuous in $(x, u)$ for a.a. $s \in[0, T]$ and continuous in $x$ uniformly in $u$, for all $h>0$ and all $|x|,|u| \leq h$ there exists a Riemann integrable function $b_{2 h}(s)$ such that $\left|g_{x}^{\prime}(s, x, u)\right| \leq b_{2 h}(s)$.

Let us present now conditions what guarantee the existence of an optimal solution of the problem (1) - (3).

Theorem 1 Let functions $f(t, x, u)$ and $g(s, x, u)$ satisfy conditions f.1), f.2) and g.1), g.2). Let the function $f(t, x, u)$ be convex in $u$, the set $U_{a d}$ be bounded, convex and weakly* closed. Let in a certain ball $S\left(x_{u}, r\right), r>0$, the linearized homogeneous equation $z(t)=$ $\int_{0}^{t} g_{x}^{\prime}\left(s, x_{u}(s), u(s)\right) z(s) d s$ have only the trivial solution $z(t)=0, t \in[0, T]$. Then optimal control and trajectory $(u, x)$ exist in $L^{\infty}([0, T]) \times C([0, T])$. 
The proof of the theorem relies on the Banach fixed point theorem. Using this theorem, we can show that the differential equation (2) (more concretely, its integral equivalent $\left.x(t)=\int_{0}^{t} g(s, x(s), u(s)) d s+x_{0}\right)$ has for a fixed admissible control $u(t) \in U_{a d}$ the solution in $C([0, T])$. Then, relying on the Weierstrass existence theorem, it is not difficult to present conditions, what guarantee the existence of optimal control and trajectories.

\section{DISCRETIZATION OF THE PROBLEM}

In order to approximate the control problem (1) - (3) by a sequence of finite dimensional problems and to analyze stability of such approximations, we should define the discrete convergence in $L^{\infty}([0, T])$. We start from a convergent quadrature process. Let

$\sum_{i=1}^{n} h\left(t_{i n}\right) \Delta t_{i n} \rightarrow \int_{0}^{T} h(t) d t, \quad n \in N$,

for any continuous on $[0, T]$ function $h(t), h \in C([0, T])$.

Convergent quadrature process (5) defines us the simpliest system of connection operators $\mathcal{P}=\left\{p_{n}\right\}$ from $C([0, T])$ to $R^{n}$ :

$\left(p_{n} x\right)_{\text {in }}=x\left(t_{i n}\right), i=1, \ldots, n, n \in N$.

Note that the connection system (6) is also applicable for the Riemann integrable functions. For Lebesgue $L^{p}$-spaces, $1 \leq p \leq \infty$, we are forced to define the system of linear connection operators $\mathcal{Q}=\left\{q_{n}\right\}, q_{n}: L^{\bar{p}}([0, T]) \rightarrow R^{n}$, only in the integral form:

$\left(q_{n} u\right)_{i n}=\Delta t_{i n}^{-1} \int_{t_{i-1, n}}^{t_{i n}} u(t) d t$

with $0=t_{0 n}<t_{1 n}<\ldots<t_{n n}=T$ and $\Delta t_{i n}=t_{i n}-t_{i-1, n}, \max _{i} \triangle t_{i n} \rightarrow 0, n \in N$.

Defined in such a way connection system satisfies the norm consistency property:

$\left\|q_{n} u\right\|_{n} \rightarrow\|u\|, n \in N, \forall u \in L^{p}([0, T]), 1 \leq p \leq \infty$

(here $\|\cdot\|_{n}$ denotes the Euclidean norm of a vector). For the $L^{p}$-spaces, $1 \leq p<\infty$, the proof of the convergence (8) relies on the fact that $C([0 . T])$ is dense in these spaces. For the space $L^{\infty}([0, T])$ the proof of the convergence (8) is presented in (Lepp, 1988).

Define the $\mathcal{Q}$-convergence (or in other words - the discrete convergence) of the sequence of vectors $\left\{u_{n}\right\}$ to the function $u(t), u \in L^{\infty}([0, T])$, in the following way.

Definition 1 The sequence $\left\{u_{n}\right\} \quad \mathcal{Q}$-converges to the function $u \in L^{\infty}$ if

$\left\|u_{n}-q_{n} u\right\|_{n} \rightarrow 0, n \in N$. 
Remark 1 The consistency property (8) guarantees the nondegeneracy of norms of discretized in $R^{n}, n \in N$, elements of $L^{\infty}$ and thus, uniqueness of the limit process (9).

In this paper we together with the $\mathcal{Q}$-convergence of vectors need also their discrete weak ${ }^{*}$ convergence. Let $z(t)$ be an integrable function, $z \in L^{1}([0, T])$.

Definition 2 The sequence $\left\{u_{n}\right\} w^{*} \mathcal{Q}$-converges to the function $u \in L^{\infty}$ if

$\sum_{i=1}^{n}\left(\left(q_{n} z\right)_{i n}, u_{i n}\right) \triangle t_{\text {in }} \rightarrow \int_{0}^{T}(z(t), u(t)) d t, n \in N \quad \forall z \in L^{1}([0, T])$.

Remark 2 The consistency property (8) holds also for any $z \in L^{1}([0, T])$.

Denote for brevity sums $f_{n}\left(x_{n}, u_{n}\right)=\sum_{i=1}^{n} f\left(t_{i n}, x_{i n}, u_{i n}\right) \triangle t_{i n}$ and $g_{n}\left(x_{n}, u_{n}\right)=\sum_{j=1}^{i} g\left(s_{j n}, x_{j n}, u_{j n}\right) \triangle t_{j n}$. Formulate the discretized problem: Minimize

$\sum_{i=1}^{n} f\left(t_{i n}, x_{i n}, u_{i n}\right) \triangle t_{i n}$

over the set of vectors $\left\{\left(x_{n}, u_{n}\right)\right\}$, satisfying constraints

$x_{i n}=\sum_{j=1}^{i} g\left(s_{j n}, x_{j n}, u_{j n}\right) \triangle t_{j n}+x_{0 n}, i=1, \ldots, n$,

and

$u_{\text {in }} \in U_{\text {nad }}, i=1, \ldots, n$.

In order to apply in approximation the existence theorem scheme, we need conditions what guarantee the discrete approximation of the integral equation (2) by the sequence of sums (11).

Proposition 1 Let the function $g(s, x, u)$ satisfy conditions $g .1)$ and let quadrature process (5) converge. Then

$\left\|g_{n}\left(p_{n} x, q_{n} u\right)-p_{n} g(x, u)\right\|_{n} \rightarrow 0, n \in N$.

Proposition 2 Let the function $g(s, x, u)$ satisfy conditions g.1), g.2). Let quadrature process (5) converge. Then from the weak ${ }^{*}$ discrete convergence of discrete controls $\left\{u_{n}\right\}$ to control $u \in L^{\infty}$ it follows strong discrete convergence of a subsequence of operators $g_{n}\left(x_{n}, u_{n}\right), \quad n \in N^{\prime \prime} \in N^{\prime} \subset N$, to a continuous function $y(t)$.

Remark 3 Note that from the last convergence it does not follow the norm (strong) convergence of $\left\{u_{n}\right\}$ to $u, n \in N^{\prime \prime}$ (if, e.g. , the operator $g$ is linear in $u$, then its inverse is not bounded - (2) is the first kind equation relative to $u$ ). 
Approximate now the integral cost functional $f(x, u)$ by the sequence $\left\{f_{n}\left(x_{n}, u_{n}\right)\right\}$ of finite dimensional sums from (10).

Proposition 3 Let function $f(t, x, u)$ satisfy conditions f.1), f.2) and be convex in u. Let quadrature process.(5) converge. Then

$\lim \sup f_{n}\left(x_{n}, u_{n}\right) \leq f(x, u)$ as $\mathcal{P}-\lim x_{n}=x, \mathcal{Q}-\lim u_{n}=u, n \in N$,

$\lim \inf f_{n}\left(x_{n}, u_{n}\right) \geq f(x, u)$ as $\mathcal{P}-\lim x_{n}=x, w^{*} \mathcal{Q}-\lim u_{n}=u, n \in N$.

Remark 4 Due to limited average of the paper, we can not present proofs of propositions. Technically they are quite lengthy and sophisticated.

Assume, relying on the Theorem 1, that the initial and approximate problems have optimal solutions $\left(x^{*}, u^{*}\right)$ and $\left(x_{n}^{*}, u_{n}^{*}\right)$, respectively.

Relying on Propositions $1-3$, we can now together with Theorem 1 formulate the main result of the paper on the approximate solution of nonlinear optimal control problems.

Theorem 2 Let functions $f$ and $g$ satisfy conditions $\left.\left.\left.\left.f_{1}\right), f_{2}\right), g_{1}\right), g_{2}\right)$. Let nonempty set of admissible controls $U_{\text {ad }}$ be convex, bounded and weakly* closed in $L^{\infty}$, and function $f(t, x, u)$ be convex in $u$. Let quadrature process (5) converge and let $U_{n a d} \subseteq q_{n} U_{a d}$. Let the linearized homogeneous equation $z(t)=\int_{0}^{t} g_{x}^{\prime}\left(s, x^{*}(s), u^{*}(s)\right) z(s) d s$ have only the trivial solution $z(t)=0$. Then

1) $f_{n}^{*} \rightarrow f^{*}, n \in N$;

2) a subsequence of discrete optimal trajectories $\left\{x_{n}^{*}\right\} \quad \mathcal{P}$-converges and a subsequence of discrete optimal controls $\left\{u_{n}^{*}\right\} w^{*} \mathcal{Q}$-converges, $n \in N^{\prime}$, to the optimal trajectory and control of the initial problem (1) - (3).

Proof. By Theorem 1 optimal solutions $\left(x^{*}, u^{*}\right)$ and $\left(x_{n}^{*}, u_{n}^{*}\right)$ of both, initial and approximate problems, exist. By Proposition 2 the sequence of operators $\left\{g_{n}\left(x_{n}^{*}, u_{n}^{*}\right)\right\}$ is $\mathcal{P}$ compact. Thus, both subsequences $\left\{x_{n}^{*}\right\}$ and $\left\{g_{n}\left(x_{n}^{*}, u_{n}^{*}\right)+x_{0 n}\right\} \mathcal{P}$-converge in uniform norm sense to the same limit. Take from the sequence $\left\{u_{n}^{*}\right\}, n \in N^{\prime}$, a $w^{*} \mathcal{Q}$-convergent subsequence $\left\{u_{n}^{*}\right\}, n \in N^{\prime \prime} \in N^{\prime} \in N$. Let $w^{*} \mathcal{Q}-\lim u_{n}=v . n \in N^{\prime \prime}, v \in U_{a d}$, and let corresponding to the control $v(t)$ trajectory be $y(t)$. Then by Proposition 3

$f^{*} \leq f(y, v) \leq \lim \inf f_{n}\left(x_{n}^{*}, u_{n}^{*}\right)=\liminf f_{n}^{*}$.

Prove the opposite inequality. Consider the difference $f_{n}\left(x_{n}^{*}, u_{n}^{*}\right)-f^{*}$. Take the sequence $\left\{\left(y_{n}, q_{n} u^{*}\right)\right\}$, where $y_{n}$ is the solution of the equation $x_{n}=g_{n}\left(x_{n}, q_{n} u^{*}\right)+x_{0_{n}}$. Then

$f_{n}^{*}-f^{*}=f_{n}\left(x_{n}^{*}, u_{n}^{*}\right)-f^{*} \leq f_{n}\left(y_{n}, q_{n} u^{*}\right)-f^{*}$.

By the definition of the $\mathcal{Q}$-convergence we have $\mathcal{Q}-\lim q_{n} u^{*}=u^{*}, n \in N$. By $\mathcal{P}-$ compactness of the sequence $\left\{g_{n}\left(y_{n}, q_{n} u\right)\right\} \mathcal{P}-\lim y_{n}=x^{*}$, where $x^{*}$ is the solution of the equation (2) with the control $u^{*}$. Hence, $f_{n}^{*}-f^{*} \leq \varepsilon$ for a small $\varepsilon>0$ and all $n \geq n_{\varepsilon}$. Consequently,

$f_{n}^{*} \rightarrow f^{*}, n \in N$. 
The second statement of the theorem follows assuming in contrary.

Corollary 1 If the function $f(t, x, u)$ is strictly convex in $u$ then the whole sequence of discrete optimal solutions $\left\{\left(x_{n}^{*}, u_{n}^{*}\right)\right\}$ of problems (10)-(12) $\mathcal{P} \mathcal{Q}$-converges to the optimal solution $\left(x^{*}, u^{*}\right)$ of the initial problem (1) - (3).

Let us compare the approach presented with papers published earlier.

1. We do not assume that for each $u \in U_{a d}$ there exist a unique solution $x_{u}$ of the equation (2), as it was done in (Daniel, 1973). Relying on the Banach fixed point theorem, we present conditions what guarantee its existence.

2. We do not use the implicit function theorem approach. The implicit function theorem and its modifications need in stability analysis of an equation $F(x)=y$ the boundedness of the inverse of the differential $F^{\prime}\left(x_{0}\right)$. From the boundedness of $F^{\prime}\left(x_{0}\right)^{-1}$ it follows noncompactness of the linear operator $F^{\prime \prime}\left(x_{0}\right)$. But, assuming only pointwise convergence of operators, one can say nothing about the convergence of solutions. Thus, the strongly regular generalized equations approach (Robinson, 1980), applied in many times in nonlinear control theory (see, e.g. , (Alt and Malanowski, 1993)), is not applicable in our case. Here we use the Banach fixed point theorem, assuming that the linearized homogeneous control equation has only the trivial solution zero.

3. We do not use the subspace approximation approach, presented in (Hager, 1990) since we do not know, how to construct a system of approximating subspaces for the space of essentially bounded functions $L^{\infty}$.

4. We do not use the approach, presented in (Mordukhovich, 1978), where the measurable control was approximated by a continuous function, using the Luzin's theorem. This theorem is not applicable in numerical analysis - there is no algorithmic way, how to realize the Luzin's $C$-property for measurable functions.

5 . We do not need convexity of $g(t, x, u)$ in $u$, usually assumed in nonlinear control theory (see, e.g. , (Fattorini, 1994)). Also, as it was written in comment 2., we assume that the linearized homogeneous control equation has only the trivial solution zero.

\section{REFERENCES}

Alt, W. and Malanowski, K. (1993) The Lagrange-Newton method for nonlinear optimal control problems. Computational Optimization and Applications, 2, 77-100.

Anselone, P.M. (1971) Collectively compact approximation theory. Prentice-Hall, New Jersey.

Atkinson, K., Graham, I. and Sloan, I. (1983) Piecewise continuous collocation for integral equations. SIAM Journal on Numerical Analysis, 20, 172-86.

Daniel, J.W. (1969) On the approximate minimization of functionals. Mathematics of Computation, 23, 573-81.

Daniel, J.W. (1973) The Ritz-Galerkin method for abstract optimal control problems. SIAM Journal on Control, 11, 53-63.

Fattorini, H.O. (1994) Existence theory and the maximum principle for relaxed infinitedimensional optimal control problems. SIAM Journal on Control and Optimization, 32, 311-31. 
Hager, W.W. (1990) Multiplier methods for nonlinear optimal control. SIAM Journal on Numerical Analysis, 27, 1061-80.

Lepp, R. (1988) Discrete approximation conditions of the space of essentially bounded functions. Proceedings of the Academy of Sciences of Estonian SSR. Physics - Mathematics, 37, 204-8 (in Russian).

Mordukhovich, B.Sh. (1978) On the difference approximations of systems of optimal control. Prikladnaya matematika i mekhanika, 42, 431-40 (in Russian).

Robinson, S. (1980) Strongly regular generalized equations. Mathematics of Operations Research, 5, 43-62.

Stummel, F. (1971) Diskrete Konvergenz linearer Operatoren, I. Mathematische Annalen, 190, 45-92.

Vainikko, G. (1978) Approximative methods for nonlinear equations. Nonlinear Analysis. Theory, Methods and Applications, 2, 647-87.

Vasin, V.V. (1982) Discrete approximation and stability in extremal problems. U.S.S.R. Computational Mathematics and Mathematical Physics, 22, 57-74. 